\title{
A Pilot Study of Exercise Training to Reduce Trunk Fat in Adults With HIV- Associated Fat Redistribution
}

Ronenn Roubenoff, Lauren Weiss, Ann McDermott, Tanya Heflin, Gregory J. Cloutier, Michael Wood and Sherwood Gorbach

\begin{abstract}
Objective: Body fat redistribution ('lipodystrophy'), with gain in abdominal and trunk fat, and decline in facial and limb fat, is a newly recognized problem in patients with HIV infection that has been linked to use of HIV-1 protease inhibitors. Increased abdominal fat may predispose these patients to hypertension, diabetes, and coronary artery disease. At this time no effective treatment is available. We examined whether exercise training could reduce trunk fat in men with fat redistribution.
\end{abstract}

Design: Open-label pilot study.

Methods: Ten men with increasing abdominal girth participated in a 16 week pilot study of progressive resistance training with an aerobic component. They trained in a community health club three times per week. Total body lean and fat mass, and trunk fat mass, were assessed by dualenergy x-ray absorptiometry (DXA).

Results: After 16 weeks of exercise, strength increased for three of the four exercises tested (leg press $+13 \%[p<0.02]$, leg extension $+19 \%[p<0.03]$, seated row $+7 \%[p<0.13]$, chest press + $18 \%[\mathrm{p}<0.005])$. There was a significant decline in total body fat by $1.5 \mathrm{~kg}(=2.1$ percentage points, $\mathrm{p}<0.01)$; most of the decline in body fat occurred in trunk fat, which decreased by $1.1 \mathrm{~kg}(\mathrm{p}$ $<0.03$ ). Weight, lean mass ( $+1.1 \pm 2.6 \mathrm{~kg}, \mathrm{p}=0.23$ ), and bone mineral density measured by DXA did not change. No adverse effects were seen from the training.

Conclusions: Exercise training may reduce trunk fat mass in HIV-positive men with fat redistribution. Controls trials of this approach are warranted. 


\section{Introduction}

Recent reports indicate that patients taking highly active antiretroviral therapy (HAART) may develop a syndrome of fat redistribution, also known as lipodystrophy, with an increase in fat in the abdomen, posterior neck ('buffalo hump') and breasts, and a loss of subcutaneous fat in the face and extremities [1,2]. Because increased abdominal fat is associated with insulin resistance, hypertension, and coronary artery disease [3], this could cause significant morbidity and mortality in HIV-infected patients. Exercise can reduce weight and coronary artery disease risk factors in populations without HIV infection [4,5], and can cause weight gain in patients with AIDS wasting [6], without increasing circulating HIV RNA [7]. We therefore carried out a pilot study to examine whether a combination of progressive resistance training and aerobic training could reduce truncal fat in patients with HIV-associated lipodystrophy.

\section{Methods}

Sixteen HIV-infected men responded to advertisements and were invited to participate in the study. Inclusion criteria were documented HIV infection, participation in the Nutrition for Life cohort study, and self-reported increase in abdominal girth. Exclusion criteria included the use of anabolic steroids, growth hormone, or megestrol acetate; lack of availability to exercise three times per week for approximately $1 \mathrm{~h}$; and inability to give informed consent. Fourteen men enrolled in the 16-week study, and 10 completed it. The reasons for discontinuation were illness in three of the men (one of whom died) and difficulty with scheduling in one. All dropouts occurred within the first 6 weeks of the study, and their results are not included in this analysis. The study was approved by the Human Investigations Review Committee of Tufts University and New England Medical Center. The main outcomes were a change in total and trunk body fat, as assessed by dualenergy X-ray absorptiometry (DXA). DXA was performed at baseline, 8 weeks, and 16 weeks using a Hologic QDR2000 densitometer operating in array mode (Hologic, Waltham, MA, USA; software version 5.64A). This method has been validated against multi-compartmental methods and compared to in-vivo neutron inelastic scattering [8-10].

Patients trained three times per week at a community health club, once in classes supervised by volunteer trainers, and twice on their own. Each session began with $20 \mathrm{~min}$ of aerobic exercise on a treadmill or stationary bicycle, followed by approximately $1 \mathrm{~h}$ of resistance training to train all the major muscle groups of the legs, back, and arms at a high level of intensity, approximately $80 \%$ of 1 repetition maximum (1-RM). Strength was assessed by 1-RM, the most weight a person can lift in good form. Compliance was examined by counting the number of sessions attended. Baseline and final measurements were compared using a $t$-test or Wilcoxon rank-sum test as appropriate.

\section{Results}

Patient characteristics are shown in Table 1. The men had an average documented increase in weight of $3.1 \mathrm{~kg}$ over the previous year (range -1.1 to $+9.5 \mathrm{~kg}$ ). No changes in antiretroviral medication occurred during the study. Compliance, as assessed by the number of times patients came to the health club, averaged $77 \%$ of the 48 possible sessions (range 53-98\%). No injuries or changes in medical condition were reported in the 10 subjects who completed the study. After 16 
weeks of exercise, there was a significant decline in total body fat by $1.5 \mathrm{~kg}(=2.1$ percentage points, $P<0.01)$; most of this decline occurred in trunk fat, which decreased by $1.1 \mathrm{~kg}(P<0.03)$. Body weight, body mass index, lean mass $(+1.1 \pm 2.6 \mathrm{~kg}, P=0.23)$, and bone mineral density measured by DXA did not change.

\section{Discussion}

Fat redistribution in HIV-infected adults appears to be a common and potentially serious threat to the long-term health of these patients. Protease inhibitors seem likely to be implicated in most cases [1,2], but discontinuation of these medicines is usually not a realistic option. The results of this pilot study indicate that exercise has the potential to improve body composition in HIVassociated lipodystrophy. However, there are several limitations to this pilot study, including: (i) the small sample size; (ii) the use of a completers rather than an intent-to-treat analysis because the dropouts occurred early in the course of the study; and (iii) the absence of a control group. However, longitudinal follow-up of over 500 subjects in Boston indicates that the natural history of body composition change with HAART is a progressive increase in fat mass with lean mass [11]. The exercise prescription employed here used intensive and progressive training, but it was carried out in a community health club setting with only limited oversight by research personnel and trainers. Therefore compliance, although good (77\%), was substantially lower than that seen in our laboratory-based studies (91\%). This no doubt explains the lower amount of strength gain in this study compared with our previous study in this population [6]. Nevertheless, the intervention had the desired effects on body com-3. position, as fat was lost without an accompanying loss of lean mass. On the basis of the results of this study, 4. further investigation of the role of exercise training in treating and preventing HIV-associated fat redistribution is warranted.

\section{Acknowledgements}

The authors thank the many volunteer trainers who made this work feasible. 


\section{References}

1. Miller KD, Jones E, Yanovski JA, Shankar R, Feuerstein I, Falloon J. Visceral abdominal-fat accumulation associated with use of indinavir. Lancet 1998, 351:871-875.

2. Lo JC, Mulligan K, Tai VW, Algren H, Schambelan M. 'Buffalo 11. hump' in men with HIV-1 infection. Lancet 1998, 351:867-870.

3. Bjorntorp P. The associations between obesity, adipose tissue distribution and disease. Acta Med Scand 1988, 723 (Suppl.):121-134. Fox AA, Thompson JL, Butterfield GE,

4. Gylfadottir U, Moynihan S, Spiller G. Effects of diet and exercise on common cardiovascular disease risk factors in moderately obese older women. Am J Clin Nutr 1996, 63:225-233.

5. Katzel LI, Bleecker ER, Colman EG, Rogus EM, Sorkin JD, Goldberg AP. Effects of weight loss vs. aerobic exercise training on risk factors for coronary disease in healthy, obese, middle-aged and older men. JAMA 1995, 274:1915-1921.

6. Roubenoff R, McDermott A, Weiss L, et al. Short-term progressive resistance training increases strength and lean body mass in adults infected with human immunodeficiency virus. AIDS 1999, 13:231-239.

7. Roubenoff R, Skolnik PR, Shevitz AH, Snydman L, Wang A, Melanson S, Gorbach S. The effect of a single bout of acute exercise on plasma human immunodeficiency virus RNA levels. J Appl Physiol 1999, 86:1197-1201.

8. Clasey JL, Hartman ML, Kanaley J, Wideman L, Teates CD, Bouchard C, Weltman A. Body composition by DEXA in older adults: accuracy and influence of scan mode. Med Sci Sports Exerc 1997, 29:560-567.

9. Economos CD, Nelson ME, Fiatarone MA, et al. A multi-center comparison of dual energy X-ray absorptiometers: in vivo and in vitro soft tissue measurement. Eur J Clin Nutr 1997, 51:312317.

10. Roubenoff R, Rall LC, Veldhuis JD, et al. The relationship between growth hormone kinetics and sarcopenia in postmenopausal women: the role of fat mass and leptin. J Clin Endocrinol Metab 1998, 83:1502-1506.

11. Silva M, Skolnik PR, Gorbach SL, et al. The effect of protease inhibitors on weight and body composition in HIV-infected patients. AIDS 1998, 12:1645-1651. 


\section{Tables}

Table 1: Characteristics of patients at baseline and after 16 weeks of training

\begin{tabular}{lccc}
\hline Characteristic & Baseline & Final & $P$ Value \\
\hline Age, years (range) & $39.2(23-56)$ & - & - \\
Ethnicity (W : B) & $8: 2$ & - & \\
HIV risk factor & & & \\
$\quad$ Homosexual contact & 7 & 9 & - \\
$\quad$ Intravenous drug use & 3 & & \\
Anti-retroviral therapy & 9 & & \\
$\quad$ Indinavir & 2 & & - \\
$\quad$ Saquinavir + Ritonavir & 3 & - & - \\
$\quad$ Nelfinavir & 4 & $24.6(3.1)$ & 0.63 \\
CD4 cell count (range) & $339(75-622)$ & & \\
HIV RNA (range) & $24.8(2.8)$ & $250(38)$ & 0.02 \\
Body mass index, kg/m ${ }^{2}$ & & $62(11)$ & 0.03 \\
Strenght, 1-RM & $220(41)$ & $41(5)$ & 0.13 \\
$\quad$ Leg press, kg & $52(16)$ & $37(4)$ & 0.005 \\
$\quad$ Leg extension, psi & $39(6)$ & $58.4(5.9)$ & 0.23 \\
$\quad$ Seated row, kg & $31(4)$ & $14.4(8.1)$ & 0.04 \\
$\quad$ Chest press, kg & $57.3(5.4)$ & $18.3(7.9)$ & 0.01 \\
Lean body mass, kg & $16.0(7.2)$ & $5.5(4.1)$ & 0.03 \\
Body fat, kg & $20.4(7.4)$ & $52.2(7.0)$ & 0.07 \\
$\quad$ as \% of body weight & $8.6(3.8)$ & &
\end{tabular}

Data are mean (SD) unless otherwise noted. 1-RM, 1 repetition maximum 